Wir gedenken das Studium der Bindung des Schwefels durch den Kautschuk noch weiter zu verfolgen und uns dabei der Vulkanisation durch ultraviolette Strahlen zu be- dienen. Wir hoffen dabei weitere Tatsachen aufzufinden, welche uns in der Feststellung der tatsächlichen Verhältnisse bei diesem ziemlich verwickelten Vulkanisationsproblem leiten können.

\title{
Beiträge zur Kenntnis der Seifen.
}

Von A. Reychler.

(Elagegangen an 28. Marz 1913)

\section{Ueber das Natriumpalmitat.}

1. Die Kristallisation der sauren Palmitate, die Mutterlaugen.

Bei der freiwilligen Abkühlung von etwa $100 \mathrm{ccm}$ einer verdünnten $(0,05$ bis $0,0025 \mathrm{n})$ Natriumpalmitatlösung bemerkt man alsbald, dab man $z u$ unterscheiden hat zwischen der Anfangstemperatur der Kristallbildung und der eigentlichen Schnellkristallisationsperiode. Erstere liegt nicht weit unter $50^{\circ}$, ist jedoch nicht immer deutlich erkennbar. Letztere nimmt nur einige Temperaturgrade in Anspruch, und iegt um so niedriger, je verdünnter die Lösung.

\begin{tabular}{|c|c|c|}
\hline $\begin{array}{l}\text { Normalität } \\
\text { der Losung }\end{array}$ & $\begin{array}{c}\text { Erste } \\
\text { Kristallbildung }\end{array}$ & $\begin{array}{c}\text { Schnell- } \\
\text { kristallisation }\end{array}$ \\
\hline $\begin{array}{c}0,05 \text { bis } 0,025 \\
0,02 \\
0,02 \text { bis } 0,004 \\
0,0025\end{array}$ & $\begin{array}{c}47 \quad 45^{\circ} \\
42,5^{\circ} \\
\text { undeutlich } \\
\text { undeutlich }\end{array}$ & $\begin{array}{r}43-42^{\circ} \\
41-40^{\circ} \\
41-37^{\circ} \\
\text { undeutlich }\end{array}$ \\
\hline
\end{tabular}

Die Beobachtungen können schon mit bloßem Auge gemacht werden, finden aber durch die Messung der (bei der Schnellkristallisation rasch abnehmenden) elektrischen Leitfahigkeit eine willkommene Bestătigung. Die bei $70^{\circ}$ sehr verschiedenen spezifischen Leitfähigkeiten erreichen bei $25^{\circ}$ den gemeinschaftlichen Wert von nur etwa 0,000250 , so dab die weitgehende Vollständigkeit der Stoffausscheidungen und die große Aehnlichkeit der Mutterlaugen deutlich hervortritt.

Schon aus den Angaben von F, Kraff $t$ über den Natriumgehalt der ausgeschiedenen sauren Seifen, zusammen mit der Behauptung, da $B$ die Mutterlaugen nur. äußerst unbedeutende Säuremengen zurückhalten, kann berechnet werden, dab die von $1 \mathrm{~g}$ Natriumpalmitat und 300 bis $900 \mathrm{~g}$ Wasser herrührenden Mutterlaugen stets ungefähr $0,002 \mathrm{n}$ Natronlösungen darstellen.

Zu fast derselben SchluBfolgerung bin ich geleitet worden durch eine Reihe von Versuchen, bei denen die beiden Phasen der bei $17^{\circ}$ auskristallisierten Systemen vollständig analysiert wurden. Beim Gebrauch von $75 \mathrm{~g}$ Wasser aut $1 \mathrm{~g}$ Palmitat war die $\mathrm{NaOH}-\mathrm{Normalitat}$ der Mutterlauge ungefahr 0,004 ; für $120 \mathrm{~g}$ Wasser sank diese Normalităt auf 0,0028 , und für 200 bis $850 \mathrm{~g}$ Wasser betrug sie 0,0018 bis 0,00175 . Der Säuregehalt der flüssigen Phase war jedoch nicht $\mathrm{zu}$ vernachlässigen, sondern erreichte durchschnittlich die Normalität 0,0003 .

2. Die Extraktion der wasserigen Seifenlösungen mittels Toluol und die Theorie der Entfettung.

Die Alkalinität der Seifenlaugen bekundet schon die hydrolytische Zerlegbarkeit der aufgelösten salzartigen Körper; und auf letzterer Eigenschaft beruht die Extrahierbarkeit der vorhandenen Säure mittels Toluol. Schon F. Krafft hat gezeigt, daB man durch wiederholte Behandlung mit diesem Lösungsmittel eine warme Natriumpalmitatlauge fast ihres ganzen Säuregehaltes berauben kann. - in qualitativer Hinsicht habe ich gefunden, daß eine Seifenlösung über ihre Kristallisationstemperatur erwärmt werden muß, um schaumfähig und extrahierbar zu werden. Und durch weitere Versuche haise ich die Extraktion von Natriumo le at lösungen bei gewöhnlicher Temperatur einem genaueren Studium unterworfen. Es wurden dazu horizontalliegende Röhren verwendet. in welchen je $50 \mathrm{ccm}$ Seifenlauge und 50 bis $25 \mathrm{ccrn}$ Toluol mit breiter Kontaktfläche längere Zeit aufeinander zu liegen kamen 1); die dabei erhaltenen Resultate lassen sich folgenderweise zusammenfassen. Bei der Anwendung gleicher Volumina der beiden Phasen war das Prozent der Extraktion dem Natrongehalt der wässerigen Lösung umgekehrt proportional (betrug also 4, 8, 16 oder 32 Proz. der anwesenden Säure, je nachdem in dex Wasserphase 3,335 oder 1,667 oder 0,834 oder 0,417 Millimol $\mathrm{NaOH}$ enthalten waren).

4) Acht Tage lang, unter hàufiger Kontakterneuerung aber sorgfaltiger Vermeidung einer jeglichen Schaumbildung. 
Beim Gebrauch von nur 40,30 oder $25 \mathrm{ccm}$ Toluol war die Extraktion nur wenig geringer.

$\mathrm{Da}$ nun das Tolunl nicht als einziges Auflossungs- und Extraktionsmittel dasteht, sondern auch andere Stoffe (nämlich die natürichen Fette) die Eigenschaft besitzen, unter günstigen Temperaturbedingungen die höheren Fettsäuren in sich aufzunehmen, so kommen wir zur folgenden Erklärung der häuslichen und industriellen Entfettungsverfahren.

Wenn ein auf Wolle ausgebreitetes Neutralfett ${ }^{2}$ ) mit einer Natriumoleatlösung in innige Berülirung kommt (Reiben und Klotzen wirken dabei sehr günstig!), so verhält es sich wie ein Extraktionsmittel und entnimmt der Lauge ein geringes Quantum Säure. Die dabei ranzig gewordenen Fettkügelchen oder Fädchen sind sonach mit Säurefunktionen umkleidet, kraft welcher sie mit der alkalischen Lauge vielerlei Beziehungen unterhalten können (Salzbildung, Dissoziation, Hydrolyse). Und da die Affinitätswirkungen des alkalischen Mediums allerseits dieselben sind, so werden die Kügelchen auseinander gehalten und am Zusammenlaufen verhindert; sie bleiben in der Flüssigkeit emulgiert. Wenn das wegzunehmende Fett nicht neutral ist, sondern von vornherein ranzig, dann wird die Theorie des Entfettungsvorganges selbstverständlich noch einfacher als wie vorher.

Wir sagen demnach mit M. E. Chevreul und F. Krafft, dab in der Wollwäscherei das den Fasern anhaftende Fett einfach in Emulsion gebracht wird und nicht durch das Seifenalkali saponifiziert $z u$ werden braucht ${ }^{3}$ ).

\section{Ueber die Cetylsulfonsäure.}

1. Darstellung und chemische Eigenschafter.

Zur Darstellung der Säure wird das Cetylsulfhydrat (aus käuflichem $\mathrm{C}_{16} \mathrm{H}_{3 g}$ I und alkoholischer NaSH-Lösung erhältlich) mit heißer Kaliumpermanganatlösung oxydiert. Aus der heib abfiltrierten $\mathrm{C}_{16} \mathrm{H}_{33} \mathrm{SO}_{3} \dot{\mathrm{K}}$-Lösung wird mit Bleiazetat das unlösliche Bleisalz gefällt, und letzteres wird in alkoholischer Suspension mit $\mathrm{H}_{2} \mathrm{~S}$ behandelt. Durch Eindampfen der alkoholischen Lösung wird die freie Säure erhalten. - Zur Reinigung kann man das Rohprodukt in heiBem

q) Odet auch Petroleum, Vaseline usw.

8) Literatur: F. Krafft, Ber.d.Deutsch.chem.Ges. 27 1747,$1755 ; 28,2566,2573 ; 29,1328,1334 ; 32,1584$, 1596 und 1608 . … A. Rey chler, Eighth Internat. Congr. of applied Chemistry 22, 221. Bull. soc. chim. de Belgique 26, 193 et 485 . Vlaamsch Congres, Leuven 1912 (Verhandelingen, Bladz. 69).
Wasser auflósen und mit $\mathrm{NaOH}$ genau neutralisieren; das bei der Abkühlung abgesetzte Natriumsalz durch Dekantation von der Mutterlauge befreien und aus wasserhaltigem Alkohol umkristallisieren, das gereinigte Salz durch Wiederholung des Bleisalzverfahrens auf freie Säure verarbeiten. Aber auch dieses Endprodukt wird, selbst in Vakuurn, nicht ganz wasserfrei. Sein Säurewert ist aber durch Titration (Phenolphthalein oder Lackmus als Indikator) sehr genau bestimmbar, und liegt stets in der Näbe von 90 Proz.

Die freie Säure ist farblos und von seifenartiger Konsistenz. Mit kaltem Wasser bildet sie homogen aussehende Gallerten oder trübe Flüssigkeiten. Mit heißem Wasser gibt sie hellere Lösungen, welche beim Abkühlen gallertartig erstarren oder sich nur mehr oder weniger trüben. Beim Bewegen der Trübungen macht sich oft ein Schimmern bemerkbar, das wohl von kristallisiertar Säure herrühren dürfte.

Aus wässeriger Lösung ist die Substanz mittels $\mathrm{H}_{2} \mathrm{SO}_{4}$ oder $\mathrm{HCl}$ aussäuerbar; es bildet sich eine ölige Schicht, welche bei etwa $44^{\circ}$ gallertartig erstart.

Die Cetylsulfonsäure ist übrigens auch löslich in Alkohol, Aether, Benzol usw. Aus Essigsäure kristallisiert sie ziemlich leicht in kleinen Lamellen.

Das Natriumsalz ist in heißem Wasser sehr löslich, scheidet sich aber beim Erkalten fast vollstandig ab (halb gallertartig). Aus wasserhaltigem Alkohol kristallisiert es sehr ausgiebig in glänzenden Lamellen. Irgendwelche Tendenz zur Bildung eines sauren Salzes ist nicht zu bemerken.

Das Bariumsalz und das Bleisalz sind in Wasser unlöslich und aus den Lösungen des gereinigten Natriumsalzes äuBerst leicht und quantitativ ausfällbar.

2. Der Seifencharakter der Cetylsulfonsäure und ihres Natriumsalzes.

In dieser Hinsicht kann folgendes gesagt werden.

1. Daß die verdünnten Lösungen der Säure, sowie des Salzes, beim Umrühren oder Schütteln leicht einen beständigen Schaum bilden.

2. Daß sie, ebenso wie die Seifenlösungen, an organische Extraktionsmittel einen Teil ihres Säuregehaltes abgeben können.

$100 \mathrm{ccm}$ einer einprozentigen Lösung von Cetylsulfonsäure in Toluol wurden vorsichtig mit $100 \mathrm{ccm}$ Wasser unterschichtet. Das System wurde sodann etwa drei Stunden bei der 
Temperatur von $40^{\circ}$ bis $50^{\circ}$ ruhig stehen gelassen, und schlieBlich (nach Wiederaufklärung der anfangs trübe gewordenen Phasen) wurde der gröBte Teil der Toluollösung isoliert und auf seinen Säuregehalt geprüft. Verdampfungsrückstand von $90 \mathrm{ccm}$ dieser Lösung wog $0,0045 \mathrm{~g}$ und konnte durch $0,15 \mathrm{ccm}$ zehntelnormaler Kalilauge genau neutralisiert werden. Das Toluol hatte demnach 0,5 Proz. der vorhandenen Säure zurückgehalten (oder extrahiert). Die Lobsungen des Natriumsulfonats sind ebenso wie diejenigen der freien Säure nur wenig extrahierbar. In keinem Falle jedoch wurde der Säuregehalt der Toluolphase gleich Null befunden.

3. soll hier noch hinzugefügt werden, daß die Entfettungskraft der besprochenen Substanzen durch direkte Versuche dargetan wurde. Beim Behandeln von roher Schweißwolle oder von fettem Wollgarn mit verdünnten Lösungen der Cetylsulfonsäure oder des Natriumsalzes, wurden stets reichliche Schaumbildung und gründliche Entfettung beobachtet.

3. Ebullioskopisches.

In einem Landsberger'schen Apparat wurden Lösungen von $\mathrm{C}_{16} \mathrm{H}_{33} \mathrm{SO}_{3} \mathrm{H}$, von $\mathrm{C}_{16} \mathrm{H}_{33} \mathrm{SO}_{3} \mathrm{Na}$ und auch von Natriumpalmitat in bekannter Weise zum Sieden gebracht. Es entwickelte sich in allen Fällen ein grobblasiger Schaum, der nicht nur das innere Rohr des Apparates, sondern auch (teilweise) den Außenraum und schlieBlich das Ableitungsrohr erfülte, so daß der Dampf nur stobweise hinausgepufft werden konnte. Dabei kam die Quecksilbersäule des Thermometers niemals zur Ruhe, da jeder Dampfstob ein Sinken und Wiederautsteigen zur Folge hatte; die wirkliche Siedetemperatur konnte jedoch aus den 0,02 bis 0,04 gradigen Oszillationen als Mittelpunkt herausgelesen werden. -- Infolge des Ueberschäumens muBte nach jeder Beobachtung die Konzentration der betreffenden Lösung tirrimetrisch odergewichtsanalytisch ermittelt werden. Stets wurde auch der Siedepunkt des reinen Wassers von neuem bestimmt. - Die erhaltenen Resultate sind in der Tabelle a zusammen. gestellt.

Es spricht von selbst, dab die berechneten Molekulargewichte $\left(M=\frac{5,2 \mathrm{p}}{d}\right)$ falsch sind und dab die Siedepunktserhöhungen $d$ in keiner Beziehung stehen zu den $p$-Werten. Diese Erhöhungen werden nur zum allergeringsten Teile durch die gelösten Körper bewirkt, rühren aber daher, daB die Schaumlamellen, in zahlreicher Folge, ihre Konkavitäten gegen die siedende Flüssigkeit richten und auf den entweichenden Dampf einen kräftigen Gegendruck ausüben. Aus den gefundenen $\Delta$-Werten und dem $\mathrm{dp}$ des Wasserdampfes bei $100^{\circ}$, kann dieser Gegendruck auf etwa $2,5 \mathrm{~mm}$ Quecksilber geschätzt werden.

Tabelle a

\begin{tabular}{|c|c|c|c|c|c|c|}
\hline $\begin{array}{c}\text { Ge- } \\
\text { brauchte } \\
\text { Sub- } \\
\text { stanz- } \\
\text { menge } \\
\end{array}$ & $\begin{array}{c}\text { Sie- } \\
\text { dende } \\
\text { loosung } \\
\mathrm{g}\end{array}$ & $\begin{array}{c}\text { Sub- } \\
\text { stanz } \\
\text { in der } \\
\text { Lösung } \\
g \\
\end{array}$ & $\begin{array}{c}\text { Nor- } \\
\text { malităt } \\
\text { der } \\
\text { Losung } \\
\mathbf{n} \\
\end{array}$ & $p$ & $\begin{array}{c}A \\
\text { Orad }\end{array}$ & $M$ \\
\hline
\end{tabular}

Cetylsulfonsäure

\begin{tabular}{l|r|r|r|r|r|r}
1,0 & 9,0 & 0,393 & 0,143 & 4,566 & 0,12 & 198 \\
0,75 & 9,48 & 0,372 & 0,128 & 4,084 & 0,11 & 193 \\
0,44 & 10,76 & 0,212 & 0,065 & 2,010 & 0,10 & 104 \\
0,48 & 14,45 & 0,302 & 0,069 & 2,135 & 0,10 & 111 \\
0,105 & 9,42 & 0,045 & 0,016 & 0,480 & 0,09 & 28 \\
0,10 & 7,54 & 0,039 & 0,017 & 0,520 & 0,11 & 25
\end{tabular}

Natriumcetylsulfonat

\begin{tabular}{l|l|l|l|l|l|l|l}
0,81 & 7,46 & 0,340 & 0,139 & 4,78 & 0,09 & 276
\end{tabular}

\begin{tabular}{l|l|l|l|l|l|l}
0,10 & 9,18 & 0,039 & 0,013 & 0,43 & 0,09 & 25
\end{tabular}

Natriumpalmitat

4. Die elektrische Leitfähigkeit von Natriumcetylsulfonatlösungen.

Die elektrolyische Zelle bestand aus einem $150 \mathrm{ccm}$ fassenden Jenaglas und einem Deckel, in welchem zwei vertikale Elektroden und ein Thermometer befestigt waren. Mit $100 \mathrm{ccm}$ Flüssigkeit beschickt hatte sie eine konstante "Kapazität". - Die zu untersuchenden Sulfonatlösungen wurden in der Zele aul $70^{\circ}$ erwärmt, der freiwilligen Abkühlung überlassen und von Zeit zu Zeit auf ihre Leitfähigkeit geprüft. $\mathrm{Da}$ die Abkühlung nur etwa sechs Slunden in Anspruch nahm, sind bei unserer Arbeitsweise Hysteresiseffekte nicht ausgeschlossen; die Leitfähigkeiten sind wohl etwas zu hoch und dic Kristallisationstemperaturen $z u$ niedrig gefunden. Die Resultate sind aber unter sich vergleichbar.

Betreffs der Tabelle $b$, weiche die spezifischen Leitfähigkeiten zum Ausdruck bringt, ist zu bemerken:

1. daß bei gewissen Temperaturen $\left(38^{\circ}\right.$ fïr die erste Konzentration, 36,5 bis $35^{\circ}$ für die zweite, und 35 bis $33,5^{\circ}$ für die dritte) die Leitfähigkeit rasch abnimmt und Schnellkristallisation andeutel, und 
2. dab die geringe Leltfăhigkeit der Mutterlaugen bei gegebener Temperatur einen fast konstanten Wert annimint. (Auf einem Temperatur-Leitfähigkeitsdiagramm würden die verschiedenen Kurven einerseits zusammenlaufen.)

Etwas Aehnliches haben wir beim Studium des Palmitats hervorgehoben. Nur waren damals die auskristallisierenden Seifen mehr oder weniger sauer und die Mutterlaugen alkalisch, während im vorliegenden Falle die beiden Phasen der abgekühlten Systeme neutral sind.

Tabelleb (Natriumcetylşulfonat)

Spezifische Leitfähigkeit $\lambda \times 10^{6}$

\begin{tabular}{|c|c|c|c|}
\hline Grad & $\begin{array}{c}0,0666 \mathrm{n} \\
\text { Losung }\end{array}$ & $\begin{array}{c}0,0333 \mathrm{n} \\
\text { Losung }\end{array}$ & $\begin{array}{c}0,01665 \mathrm{n} \\
\text { Losung }\end{array}$ \\
\hline $\begin{array}{l}65 \\
60 \\
55 \\
50 \\
45\end{array}$ & $\begin{array}{l}3000 \\
2764 \\
2576 \\
2375 \\
2151\end{array}$ & $\begin{array}{l}1535 \\
1403 \\
1303 \\
1184 \\
1086\end{array}$ & $\begin{array}{l}860 \\
786 \\
712 \\
651 \\
582\end{array}$ \\
\hline 40 & $1966\left\{\begin{array}{l}\text { Krist. } \\
\text { Strelfem aut } \\
\text { dem Boden } \\
\text { des OefäBes }\end{array}\right.$ & 981 & 524 \\
\hline 39 & 1900 (2.Elektrode & - Krist. & 518 Kristall- \\
\hline 37 & 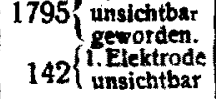 & $\begin{array}{l}940\left\{\begin{array}{l}\text { Streifen auf } \\
\text { dem Boden }\end{array}\right. \\
912\end{array}$ & 496 $\left\{\begin{array}{l}\text { streifen auf } \\
\text { dem Boden } \\
\text { und den } \\
\text { seitenwänd. }\end{array}\right.$ \\
\hline 36 & 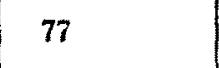 & $829\left\{\begin{array}{l}\text { Elektroden } \\
\text { unsichtbar } \\
\text { geworden }\end{array}\right.$ & 480 \\
\hline 35,5 & & 240 & - f 2. Elektrode \\
\hline 35 & 69 Bodenabs. & 120 & 438 maskiert \\
\hline 34,5 & - & 86 & 253 mastriert \\
\hline $\begin{array}{l}34 \\
33,5 \\
33 \\
30\end{array}$ & $\frac{62}{58}$ & $\begin{array}{l}79 \\
71 \\
58\end{array}$ & $\begin{array}{l}104 \\
74 \\
68 \\
53\end{array}$ \\
\hline 24 & - & - Bodensatz & $37\left\{\begin{array}{l}\text { 1. Eichtbar } \\
\text { sicher }\end{array}\right.$ \\
\hline 22 & 32 & $38\left\{\begin{array}{c}\text { Die 1. Elek- } \\
\text { trode vied. } \\
\text { sich tbar }\end{array}\right.$ & - \\
\hline 20 & - & 一 & 32 Bodensatz \\
\hline $\begin{array}{l}18,5 \\
17,5\end{array}$ & $26\left\{\begin{array}{c}\text { Bedentend. } \\
\text { Bodensatz }\end{array}\right.$ & - 30 & - \\
\hline
\end{tabular}

Tabelle c (Natriumcetylsulfonat) Molekularleitfähigkeit

\begin{tabular}{c|c|c|c}
\hline \multicolumn{2}{|c|}{$\begin{array}{c}\text { Normalitat: } 0,6666 \\
\text { Verdun nung : } 15 \text { Lit. }\end{array}$} & $\begin{array}{l}0,0333 \\
30 \text { Lit. }\end{array}$ & $\begin{array}{l}0,01665 \\
60 \text { Lit. }\end{array}$ \\
\hline Temperatar orad & & & \\
65 & 45 & 46 & 51,6 \\
60 & 41,5 & 42,1 & 47,2 \\
55 & 38,6 & 39,1 & 42,7 \\
50 & $\mathbf{3 5 , 6}$ & 35,5 & 39,1 \\
$\mathbf{4 5}$ & $\mathbf{3 2 , 3}$ & 32,6 & $\mathbf{3 4 , 9}$ \\
40 & 29,5 & 29,4 & 31,4
\end{tabular}

Die Molekularleitfahigkeiten (Tabelle c) scheinen für die zwei ersten Lösungen nur wenig von der Konzentration beeinflubt $z u$ werden.

5. Die Leitfähigkeit von wässerigen Lösungen der Cetylsulfonsăure.

Erste Arbeitsweise. Die Messungen wurden ebenso ausgeführt wie beim Studium des Natriumcetylsulfonats, so dab die erhaltenen Resultate wohl untereinander vergleichbar aber wahrscheinlich mit einem systematischen Hysteresiseffekt behaftet sind.

Aus den Zahlen der Tabelle $d$ und aus den Kurven des Diagramms 1 läbt sich herleiten, dab die Gerinnung respektive Träbung der untersuchten Lösungen stets zwischen denselben Temperaturgrenzen $(24-19)^{\circ}$ eintrat, und $\mathrm{da} B$ die flüssige Phase der verschiedenen Systeme (der leitend gebliebene Anteil) bei gegebener Temperatur eine nur wenig veränderliche Leitfăhigkeit erkennen lieb.

Die Molekularlejtfähigkeiten (Tabelle e) sind natürlich nur für höhere Temperaturen berechenbar. Besonders hervorgehoben zu werden verdient der Umstand, das für unsere konzentriertesten Lösungen die $\mu$-Werte bei steigender Verdünung abnehmen.

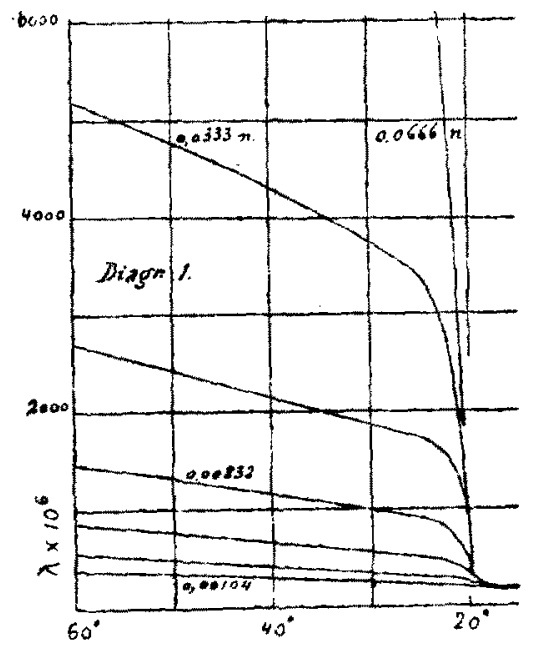

$Z$ weite Arbeitsweise. Hierbei betanden sich die zu untersuchenden Lösungen in einem Kohlrausch gefä̉i (von der Kapazität 3,09 ), welches in einem kleinen mit Azetondamp? umgebenen Wasserbad zu stehen kam. Jede Lösung verblieb etwa eine halbe Stunde in dern auf $56,2^{\circ}$ erwärmten Thermostat, und die 
Tabelled (Cetylsulfonsäure)

Spezifische Leitfähigkeit $\lambda \times 10^{6}$

\begin{tabular}{|c|c|c|c|c|c|c|c|}
\hline Normalita & 0,0666 & 0,0333 & 0,01665 & 0.00832 & 0,00416 & 0,00208 & 0,00104 \\
\hline \multicolumn{8}{|l|}{ Temperatur Orad } \\
\hline & 11116 & 5432 & 2861 & 1593 & 956 & 637 & 439 \\
\hline & 10622 & $\begin{array}{l}5196 \\
4970\end{array}$ & 2716 & 1503 & 885 & 583 & 402 \\
\hline $\begin{array}{l}50 \\
50\end{array}$ & $\begin{array}{r}10170 \\
9755\end{array}$ & $\begin{array}{l}49 / 9 \\
4780\end{array}$ & 2451 & $\begin{array}{l}1400 \\
1310\end{array}$ & $\begin{array}{l}752 \\
759\end{array}$ & $\begin{array}{l}0,00 \\
493\end{array}$ & $\begin{array}{l}311 \\
339\end{array}$ \\
\hline 45 & 9372 & 4579 & 2320 & 1226 & 703 & 451 & 308 \\
\hline 40 & 9019 & 4306 & 2173 & 1144 & 655 & 412 & 283 \\
\hline 35 & 8386 & 4051 & 2034 & 1067 & 605 & 379 & 258 \\
\hline $\begin{array}{l}30 \\
27\end{array}$ & $\stackrel{7836}{-}$ Op & 3764 & $\begin{array}{l}1882 \\
1804\end{array}$ & 996 & 556 & 346 & 238 \\
\hline $\begin{array}{l}27 \\
25\end{array}$ & 6928 & 3390 Op & 1738 & $\begin{array}{l}947 \\
912\end{array}$ & 508 & 314 & 216 \\
\hline 24 & 6373 & 3187 & 1707 Op & & $\overline{109}$ & 310 & - \\
\hline 23 & $\overline{6128}$ & 2933 & $\begin{array}{l}1648 \\
1562\end{array}$ & $\begin{array}{l}877 \\
854\end{array}$ & $\begin{array}{l}493 \\
483\end{array}$ & 301 & 204 \\
\hline 22,5 & 5690 & 2845 & 1471 & 817 & 464 & & \\
\hline 22 & $\begin{array}{c}4345 \\
2 u\end{array}$ & 2598 & 1414 & 784 & 447 & $2850 p$ & 196 \\
\hline 21,5 & - & 2124 & 1350 & 735 & 419 & - & -. \\
\hline & 2897 & 1738 & 1258 & 673 & $\begin{array}{l}2 \mathrm{n} \\
392\end{array}$ & 254 & - \\
\hline 20,5 & - & 1366 & 1017 & 613 & 354 & - & - \\
\hline 20 & 919 & 996 & 747 & 509 & 327 & 221 & $177 \mathrm{Op}$ \\
\hline & $1 \mathrm{u}$ & & $2 u$ & & & & \\
\hline 19 & 419 & 319 & 244 & 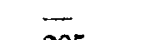 & 240 & 198 & 170 \\
\hline 18 & -- & 250 & 199 & 205 & & - & 165 \\
\hline 17 & - & . & 191 & - & 199 & - & 160 \\
\hline
\end{tabular}

NB. Abkirzungen der Tabelle $\mathrm{d}: \mathrm{Op}=$ Anfang des Opaleszierens. $2 \mathrm{n}=$ die zweite, die vom Beobachter entfernteste Elektrode ist nebelig verdeckt. $2 u=$ die zweite Elektrode ist unsichtbar geworden. $1 \mathrm{n}$ oder $\mathbf{u}$ haben Bezug auf die erste Elektrode.

Tabelle e (Cetylsulfonsarure)

Molekularleitfähigkeit

\begin{tabular}{|c|c|c|c|c|c|c|c|}
\hline \multicolumn{2}{|c|}{$\begin{array}{l}\text { Normalitat: } 0,0666 \\
\text { Verdunnung: } 15 \text { Lit. }\end{array}$} & $\begin{array}{l}0,0333 \\
30 \text { Lit. }\end{array}$ & $\begin{array}{l}0,01665 \\
60 \text { Lit. }\end{array}$ & $\begin{array}{l}0,00832 \\
120 \text { Lit. }\end{array}$ & $\begin{array}{l}0,00416 \\
240 \text { Lit. }\end{array}$ & $\begin{array}{l}0,00208 \\
480 \text { Lit. }\end{array}$ & $\begin{array}{l}0,00104 \\
960 \text { Lit. }\end{array}$ \\
\hline $\begin{array}{c}\text { Temperatur Orad } \\
60 \\
55 \\
50 \\
45 \\
40 \\
35 \\
30 \\
25\end{array}$ & $\begin{array}{l}159,3 \\
152,5 \\
146,3 \\
140,6 \\
135,3 \\
125,8 \\
117,5 \\
103,9\end{array}$ & $\begin{array}{l}155,9 \\
149,4 \\
143,4 \\
137,4 \\
129,2 \\
121,5 \\
112,9 \\
101,7\end{array}$ & $\begin{array}{l}163 \\
155 \\
147,1 \\
139,2 \\
130,4 \\
122 \\
112,9 \\
104,3\end{array}$ & $\begin{array}{l}180,4 \\
168,7 \\
157,2 \\
147,1 \\
137,3 \\
128 \\
119,5 \\
109,4\end{array}$ & $\begin{array}{l}212,4 \\
197,8 \\
182,2 \\
168,7 \\
157,2 \\
145,2 \\
133,4 \\
121,9\end{array}$ & $\begin{array}{l}279,8 \\
257,3 \\
236,6 \\
216,5 \\
197,8 \\
181,9 \\
166,1 \\
150,7\end{array}$ & $\begin{array}{l}385,9 \\
356,2 \\
325,4 \\
295,7 \\
271,7 \\
247,7 \\
228,5 \\
207,4\end{array}$ \\
\hline
\end{tabular}

Messungen wurden bis zur Koristanz wiederholt. - Alle Flüssigkeitsmessungen und Verdünnungen geschahen bei der Temperatur von $40^{\circ}$

Aus den erhaltenen Resultaten geht hervor, daß die zwei ersten Wasserzusätze (Verd. 7,78 $\rightarrow 15,56 \rightarrow 31,1)$ wirklich ein Fallen der Molekularleitfähigkeit verursachen; und da nun der Hysteresisfehler auf ein Geringes reduziert ist, so kann dieses Verhalten der Cetylsulfon- säure als erwiesen gelten ${ }^{4}$ ). Uebrigens wird man auch bemerken, daß die maximale Leitfähigkeit $\left(\mu_{\infty}\right)$, welche auf etwa 550 geschätat werden dürfte, bei unserer gröBten Verdünnung noch nicht erreicht ist.

4) Ein ähnliches Verhalten wurde für mehrere Seifen konstatiert: J. Mc. Ba in, Ber. d. Deutsch. chem. Ges. 43, 321; Zeitschr. f. physik. Chem. 76, 179; Trans. Chem. Soc. 101, 2042. F. Goldschmidt, Zeitschr. f. Elektmchem. 18, 380; Koll.-Zeitschr. 12, 18 (1913). 
Tabellef (Cetylsulfonsaure) Temperatur: $56,2^{\circ}$

\begin{tabular}{c|c|c|c|c}
\hline $\begin{array}{c}\text { vormali- } \\
\text { tät der } \\
\text { Lösungen }\end{array}$ & $\begin{array}{c}\text { Ver- } \\
\text { dünnung } \\
\text { (Liter) }\end{array}$ & $\begin{array}{c}\text { Vethaltnis } \\
\text { der Ver- } \\
\text { dünnungen }\end{array}$ & $\begin{array}{c}\text { Spezit. } \\
\text { Leit- } \\
\text { tähigkeit }\end{array}$ & $\begin{array}{c}\text { Molekul- } \\
\text { Leit- } \\
\text { tähigkeit }\end{array}$ \\
\hline 0,12854 & 7,78 & 1 & 0,021020 & 163,5 \\
0,06427 & 15,56 & 2 & 0,009196 & 143,1 \\
0,03213 & 31,1 & 4 & 0,004352 & 135,3 \\
0,01607 & 62,2 & 8 & 0,002332 & 145,1 \\
0,00803 & 124,5 & 16 & 0,001332 & 165,8 \\
0,00402 & 248,8 & 32 & 0,000803 & 199,8 \\
0,00201 & 497,5 & 64 & 0,000520 & 258,7 \\
0,001005 & 995 & 128 & 0,000365 & 363,2 \\
0,000503 & 1990 & 256 & 0,000238 & 473,6
\end{tabular}

NB. Die Zahlen der dritten Kolumne tindet man auch auf der Abszisse des Diagramms 2.

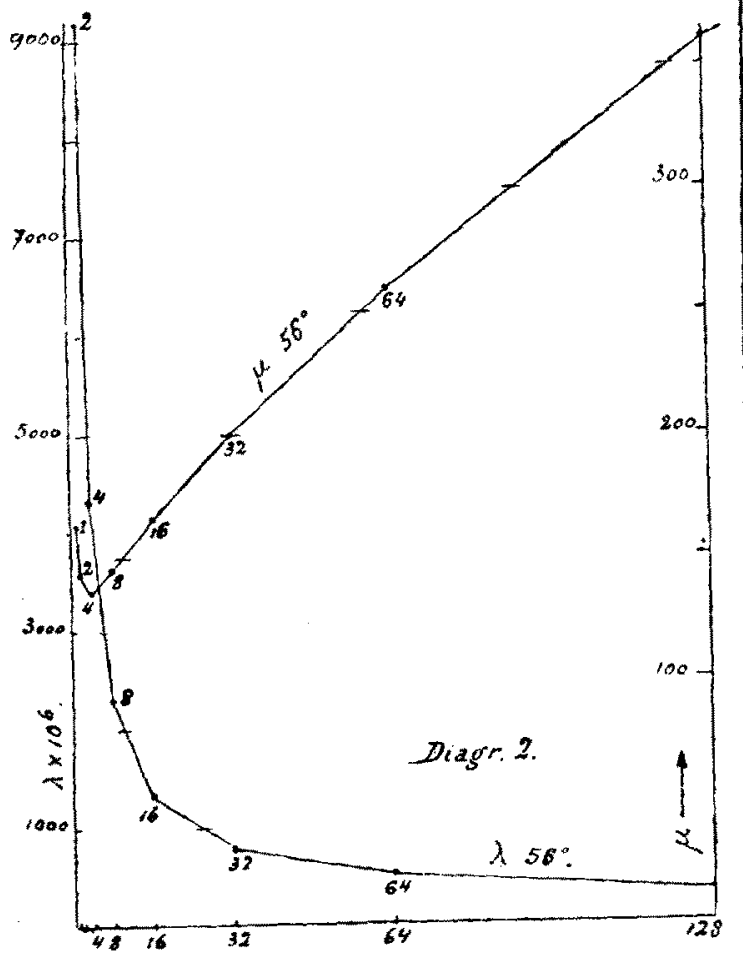

6. Schlubbetrachtungen.

Wenn man das Diagramm 1 näher ins Auge faBt, so kommt man alsbald zur folgenden Interpretation der durch die Zeichnung erimnerten Erscheinungen. Unsere Cetylsulfonsäure-Wassersysteme sind nicht innerlich homogen, sondern bestehen aus Mizellen und Intermizellarflüssigkeit. Letztere enthält ein geringes Quantum Säure wirklich gelöst, und behauptet bei allen Temperaturen ihre eigene Leitfăhigkeit. Die Mizellen dagegen sind bei niedriger Temperarur erstart und nichtleitend, bei höheter verflüssigt und leitend. Daher kommt es, dab bei einer geurissen Temperatur die Leitfähigkeit unserer Systeme plötzlich ansteigt oder sinkt, je nachdem der Uebergangspunkt im cinen oder im anderen Sinne überschritten wird. Der Sprung in den $\lambda$-Werten ist natürlich um so größer, je mizellenreicher (je konzentrierter) das betrachtete System. - Nach unserer ersten Arbeitsweise und bei sinkender Temperatur wurde der Uebergangspunkt in der Nähe von $24^{\circ}$ situiert. Aus schon bekannten Gründen ist jedoch dieser Punkt etwas höher zu verlegen. (40 bis $42^{\circ}$ ?)

Aus dem Diagramm 2 ist zu ersehen, dab die ersten Verdünnungen des anfänglichen Systems das molekulare Leitvermögen e $r$ n i edrigen, und dieser Befund ladet ebenfalls zu näherer Betrachtung ein. Es läßt sich ein System ausdenken, in weichem die Mizellen so dicht zusammengedrängt sein würden, daß ihre äuBeren Wasserhüllen zusammenflieBen, so daß von einer Art Homogenität die Rede sein könnte. Ein erster Wasserzusatz, sagen wir eine Volumdoppelung, würde sodann folgendes bewirken: a) die Produktion einer sehr bedeutenden Menge von Intermizellarflüssigkeit; b) eine geringe Verminderung der Mizellenmasse (durch Auflösung); und c) vielleicht eine Steigerung des Hydratationsgrades der Mizellen. Hinsichtlich des Leitvermögens $(\mu)$ würden $a+b$ sicher eine Vergröberung zur Folge haben, denn die Molekularleitung der Säure ist größer in der Flüssigkeit als in den Mizellen; und $c$ könte ebenfalls im positiven Sinne mitwirken. Aber auch ein negativer Einflub d würde sich geltend machen, denn durch die Verdünnung wird die Homogenität des Systems vermindert, so daß der für die E! ektrizität offenstehende Weg verwickelter und widerstandsfähiger wird als wie zuvor. Es ist jedoch zu bemerken, dafi d nur bei den ersten Verdünnungen stark negativ sein kann, und beispäteren Verdünnungen nicht nur all malich schuacherwird, weil die aufeinanderfolgenden Homogenitätsverminderungen dem vorher erreichten Inhomogenitätsgrad umgekehrt proportional sind, sondern sogar das Vorzeichen àndert, weil der schliebslich angestrebte Zustand des Systems ein solcher von allergröbter Einförmigkeit (eine mizellenfreic Lösung) ist. Durch unser Diagramm 2 wird gezeigt, dab nur bei unseren beiden ersten Verdünnungen die Effekte a, b und $c$ durch d überkompensiert werden und daB, nach dem 
Erreichen eines Minimums, die $\mu$-Werte mit fortschreitender Verdünnung regelmäBig ansteigen.

Bei unserer Verdünnung 2000 ist die Endhomogenität noch nicht erreicht, so daB die Intermizellarflüssigkeit sicher verdünnter ist als $0,0005 \mathrm{n}$. Es soll uns daher nicht wundernehmen, daß bei den ebullioskopischen Versuchen, bei welchen doch nur die Intermizellarflüssigkeit ins Sieden kơmmt, die siedepunktserhöhende Wirkung der gelösten Säure durch Nebeneinflüsse total verdeckt wird.

Wenn wir es jetzt versuchen wollen, die Kolloidnatur der Cetylsulfonsäure mit der chemischen Konstitutionsformel in Beziehung zu bringen, so kommen wir zu den folgenden Ueberlegungen. Durch die Löslichkeit und sogar die Deliqueszenz der meisten $\mathrm{C}_{n} \mathrm{H}_{2 n+1} \mathrm{SO}_{3} \mathrm{H}$ Verbindungen wird die Hydrophilie der $\mathrm{SO}_{3} \mathrm{H}$-Gruppe hinreichend erwiesen. Wenn aber die Kohlenwasserstoffkette sehr lang wird, dann ist diese nur noch teilweise durch die löslichmachende Gruppe beherrscht, so dab die bipolarlokalisierten Eigenschaften des Molekulargebäudes zur Geltung kommen können.
Einerseits sind dann die Moleküle kohlenwasserstoffartig hydrophob, und nur zur homogenen Assoziation geneigt; andererseits sind sie mit der Hydrophilie des $\mathrm{SO}_{9} \mathrm{H}$ begabt und sehr wasserbegierig. Damit nun diese verschiedenen Affinitäten möglichst Befriedigung finden, bilden sich Molekülkomplexe, in denen kohlenwasserstoffartige Kerne mit hydrophilen $\mathrm{SO}_{3} \mathrm{H}$-Funktionen, und mit einer von diesen fest angezogenen Wasserhülle umkleidet sind; mit anderen Worten, die Moleküle gruppieren sich zu Mizellen.

In konzentrierten Systemen können die Mizellen sehr dicht zusammengedrängt sein; in verdünnteren sind sie durch die Intermizellarflïssigkeit voneinander getrennt, und auch wohl einigermaßen zerfallen. Bei äußerster Verdünnung würden sie zu Einzelmolekülen zergliedert und wirklich gelöst werden. Das Leitvermögen der Mizellen und der Flüssigkeit beruht natürlich auf elektrolytische Dissoziation ${ }^{5}$ ).

Aehnliche Betrachtungen dürften auch beim Studium von anderen Kolloidsubstanzen von Nutzen sein.

5) Im Bull. de la Soc. Chim. de Belgique wird dasselbe Thema etwas ausiuhrlicher entwickelt werden

\section{Bücherbesprechungen.}

Binchemie der Haut von P. G. Unna. $105 \mathrm{~S}$. M. 3.-. (Verlag von Gustav Fischer, Jena 1913.)

Die tierische Haut ist bekanntlich durch ihten Kollagengehalt eine der Herkunitsstatien des Leims. Dem letzteren verdankt die Kolloidchemie ihren Namen. Schon deshalb erscheint eine Anzeige dieses wertvollen Werkes an dieser Stelle berechtigt.

Es $\mathrm{kam}$ dadurch zustande, daß der inmitien einer ausgedehnten dermatologischen Praxis stehende Verfasser sich sagte: Ein Verlassen der bisherigen rein empirischen Ausarbeitung der dermatologischen Therapie ist nur dann moglich, wenn wir das Medium genau kennen, auf welches wir die Heilmittel einwirken lassen. Die Kenntnis der normalen Hautchemie mub dabej natürlich derjenigen der pathologischen Zustände vorausgehen. - Mit einer bewundernswerten Elastizitat warf sich der Hamburger Arzt fir eine Reihe von Jahren auf dies Gebiet. Und da er sich nicht mit der bloßen Feststellung dieser und jener Substanzen in den einzelnen Zellarten zufrieden gab, entstand ein Werk, das uberall dem Dynamischen neben dem Deskriptiven einen breiten Raum gewshrt.

Zwar war in dieser Beziehung das Ideal noch nicht möglich. Denn man kann noch nicht die einzelnen Bausteine der Nahrung durch Blut und Lymphe bis in die einzelnen Zellen der Haut verfolgen. Man sieht noch nicht, wie cine Zellart es fertig bringt, ihren hochkomplizierten Bau durch die ankommenden Aminosäuren, Kohlehydrate und Fette unter Abspaltung der verbrauchten und ubberflussig gewordenen
Bausteine zu ergänzen. Denn in der Gleichung:
Zelle $-x=y$ fehlt zurzeit nicht nur die Kenntnis von $x$ und $y$ im allgemeinen, sondern besonders auch die genaue Kenntnis vom chemischen Bau der Zelle selbst. Nur in einem einzigen aufzunehmenden Bestandteile des Blutes, also von einem $x$, wat es durch $P$. G. Unna's Vorstudien klar geworden, welche Bestandteile inn vorzugsweise speichern, welche itm vollständig verbrauchen und welche inn an andere Elemente wieder abgeben können - das ist der Sauerstoff. An Hand des Sauerstofiwechsels versucht deshalb P. G. Un na ein Bild des Gesamtstoffwechsels der Haut zu gewinnen.

Es gelingt ihm dies mil Hilfe der Farbemethoden. Während die Histologen letztere fast ausschlieblich zur Feststellung der inneren Form der Gewebe benutzen, werden sie bei P. G. Unna zu Reagenzien der chemischen Analyse. Es war dabei ein glücklicher Zufall, daß die Entdeckung des stark reduzierenden Rongalits $(=$ Kondensationsprodukt des Formaldehyds mit dem Natriumsalz der Sulfoxylsäure) zeitlich mit dem Beginn seiner Studien fast zusammenfiel. Das aus diesem und Methylenblau hergestellte Rongatitweif erwies sich als ausgezeichnetes Mittel zur Feststellung der "Sauerstofforte" im Gewebe. In der Haut sind sie hauptsächlich in den Mastzellen und Kernen vorhanden. Spongioplasma, Muskel, Nerv und Hornschicht stellen dagegen Reduktionsorte dar.

Auf die auBerordentlich wichtigen Details dieser Untersuchung, welche auch das Studium anderer Gewebe reichlich befruchten werden, karn hier leider nicht eingegangen werden. Es sei nur auf einige 